\title{
USO DEL FUEGO Y RÉGIMEN DE INCENDIOS EN LA PROVINCIA DE CÁCERES. CONCLUSIONES A PARTIR DE LOS DATOS DEL INTERROGATORIO DE LA REAL AUDIENCIA DE EXTREMADURA (1790)
}

\author{
Cristina Montiel Molina \\ Universidad Complutense de Madrid \\ crismont@ucm.es \\ ORCID iD: https://orcid.org/0000-0003-3069-7534 \\ Luis Galiana Martín \\ Universidad Autónoma de Madrid \\ luis.galiana@uam.es \\ ORCID iD: https://orcid.org/0000-0002-8492-1637
}

Recibido: 18/01/2019; Aceptado: 05/11/2019.

\begin{abstract}
Cómo citar este artículo/Citation: Montiel Molina, C. y Galiana Martín, L. (2019). Uso del fuego y régimen de incendios en la provincia de Cáceres. Conclusiones a partir de los datos del Interrogatorio de la Real Audiencia de Extremadura (1790). Estudios Geográficos, 80 (287), e027. https://doi.org/10.3989/estgeogr.201944.024
\end{abstract}

RESUMEN: El objetivo del artículo es caracterizar y evaluar la presencia del fuego como instrumento de gestión del territorio, señalando las continuidades y cambios en su uso desde finales del siglo XVIII hasta mediados del siglo XX. A partir de la situación descrita en el Interrogatorio de la Real Audiencia de Extremadura de 1790, se realiza un análisis evolutivo del uso del fuego en la provincia de Cáceres mediante la información suministrada por fuentes judiciales y policiales depositadas en el Archivo Histórico Provincial (AHP). Se maneja además el Registro Histórico de Incendios Forestales (RHIF) creado por el grupo de investigación Complutense, que se remonta al año 1497 y cuenta con casi 7.000 registros de incendios históricos datados y documentados. Todo ello ha permitido profundizar en el conocimiento sobre la distribución espaciotemporal del fuego útil, su relación con la organización del terrazgo y los conflictos que generaba, suministrando interesantes conclusiones sobre la intensa relación existente entre su uso en el marco de los sistemas agrarios tradicionales y el posterior régimen de incendios que se instala acompañando la crisis de dicho sistema de organización.

PALABRAS CLAVE: Historia del fuego; pirotransición; transición forestal; incendios históricos; crisis rural; provincia de Cáceres.

\section{FIRE USE AND WILDFIRE REGIME IN THE PROVINCE OF CÁCERES. CONCLUSIONS FROM DATA OBTAINED IN THE INTERROGATORIO DE LA REAL AUDIENCIA DE EXTREMADURA (1790)}

ABSTRACT: This paper aims at characterizing and evaluating the use of fire as a management tool in the rural areas. The final objective is to clarify the continuities and changes in burning practices from the late eighteenth century to the mid-twentieth century. The starting point to analyse the evolution of the use of fire in the province of Cáceres has been the description presented in the Interrogatorio de la Real Audiencia de Extremadura formed in 1790, as well as the information gathered in different judicial and police sources kept in the Province Historical Archive. The Fire History Dataset developed by the Complutense Research Group and its metadata of more than 7,000 historical fire records since 1497 have also been essential. All this information has allowed deepening knowledge about the spatial and temporal patterns of fire use practices, its connection with the organization of productive agriculture land and the related conflicts. In conclusion, we have proved the strong relationship between the use of fire in the framework of traditional agrarian systems and the subsequent wildfire regime installed as a result of the rural crisis.

KEY WORDS: Fire history; pirotransition; forest transition; historic fires; rural crisis; provincia of Cáceres. 


\section{INTRODUCCIÓN}

Existe una coincidencia generalizada en destacar la importancia que el uso del fuego llegó a alcanzar en los sistemas agrarios tradicionales del mundo mediterráneo, si otorgamos esta genérica apelación a los desarrollados con anterioridad al éxodo rural masivo de mediados del siglo XX (Pyne, 2012; Pasquale, Martino y Mazzoleni, 2004). En este ámbito, el recurso a diferentes tipos de quemas ha sido consustancial a buen número de prácticas agrícolas, ganaderas y silvícolas, constituyendo una herramienta insustituible en la gestión agraria del territorio (Naveh, 1975; Lázaro y Montiel, 2010). Esta idea firmemente establecida contrasta, sin embargo, con un escaso conocimiento de cómo se utilizaba el fuego en diferentes momentos históricos y en distintos contextos territoriales. En efecto, más allá de algunas referencias generales comúnmente repetidas, desconocemos casi todo sobre su distribución espaciotemporal en relación con la organización del terrazgo y/o con las condiciones geoecológicas del terreno, así como sobre los conflictos que generaba el uso del fuego (Araque, Galiana y Montiel, 2012).

La presencia del fuego en el territorio ha respondido a dos tipos de razones a lo largo de la historia. Por un lado, a la propia expansión de la actividad agraria desde el Neolítico, para conquistar y mantener el espacio agrícola y ganadero. En el marco de los sistemas tradicionales agroganaderos, su recurso era imprescindible para abrir áreas de monte (Balboa, 1999), conseguir terrenos para cultivos temporales tras las prácticas de roza y quema, mejorando la fertilidad de suelos pobres para la agricultura, y para beneficiar el crecimiento de plantas anuales de mayor valor forrajero. El valor fertilizante de las cenizas explica la pervivencia de la quema de rastrojos herbáceos, así como de diferentes tipos de restos vegetales en los cultivos leñosos. La efectividad y economía de estas prácticas tradicionales ha hecho que algunas de ellas se hayan mantenido en la Europa meridional hasta la actualidad (Montiel y Kraus, 2010). Por otro lado, el fuego en los montes y bosques ha sido también una manifestación de conflictos estructurales y coyunturales. Su uso para ampliar y mantener los pastos, y su extensión al monte, traduce enfrentamientos ancestrales por el aprovechamiento de los recursos forestales entre pastores, propietarios de pastos y comunidades rurales (Leone, 1999). En las sierras cacereñas, la competencia entre colmeneros y los fuegos provocados por las poblaciones locales es- tán recogidas en buen número de ordenanzas y disposiciones punitivas (Berrogain, 1930; Blanco, 1994). En Extremadura, el hambre de tierras está en el origen del fuerte incremento de los incendios en los montes en el tránsito entre los siglos XIX Y $\mathrm{XX}$, directamente relacionados con la fuerte crisis del mundo rural por el crecimiento de la población y la necesidad de ampliar la superficie de cultivo (Sánchez, Araque, Pulido y Moya, 1999). Algo similar había ocurrido a finales del siglo XVIII con las ocupaciones y roturas de fincas por parte del vecindario, acompañadas de incendio, tras el Real Decreto de 1793, que declaró las dehesas extremeñas de pasto y labor (García y Sánchez, 1984).

En el largo proceso de progresivo declive y posterior recuperación de la cubierta forestal sucedido en los últimos siglos, conocido como transición forestal (Rudel, Schneider y Uriarte, 2010; Mather, 1992; Barbier, Burgess y Grainger, 2010), la presencia del fuego y su variación han jugado un papel fundamental (Iriarte-Goñi y Ayuda, 2018; Krebs, Pezzatti, Mazzoleni, Talbot y Conedera, 2010; Pausas y Keeley, 2009; Pausas y Fernández, 2012). Para valorar adecuadamente este factor, se ha argumentado la necesidad de ampliar el conocimiento del régimen de incendios a época histórica (Araque, 1999; Montiel, 2013b). Por idéntica razón, y con no menos importancia, se hace igualmente necesario caracterizar y evaluar la presencia del fuego útil. Sólo de esta manera se podrán determinar con mayor precisión su importancia como elemento regulador del combustible y su acción modeladora sobre la distribución y estado de la cubierta vegetal (Abel-Schaad y López-Sáez, 2013).

En la provincia de Cáceres confluyen circunstancias de diversa índole que han facilitado una aproximación privilegiada al problema de la presencia del fuego y su variación espaciotemporal. Por un lado, la pervivencia de prácticas agroganaderas con frecuente recurso a las quemas; y junto a ello, una alta conflictividad social en el uso de la tierra, provocadora de no pocos incendios. Al mismo tiempo, una magnífica documentación histórica desde finales del siglo XVIII hasta la actualidad. En este sentido, el Interrogatorio de la Real Audiencia de Extremadura, punto de partida de la investigación, constituye una magnífica relación territorial de la situación a finales del siglo XVIII. Su confrontación con documentación judicial y policial posterior, junto con otras fuentes secundarias, han permitido una completa caracterización de la presencia del fuego en el territorio en el marco de los sistemas agrarios tradicionales (Galiana, 2014). 


\section{MARCO TEÓRICO}

La presencia y el impacto del fuego en el territorio están directamente vinculados a la actividad humana, tanto de forma directa a través de la ignición y extinción, como de forma indirecta mediante la gestión del combustible, las medidas de prevención, y los cambios en la población y en su comportamiento (Turco et al., 2016). Esta estrecha interacción entre los factores humanos y el régimen del fuego ${ }^{1}$ ha evolucionado además a lo largo de la historia en un complejo diálogo que ha atravesado por situaciones de crisis y ruptura, desestabilizando el sistema y dando lugar a una evolución no lineal de los incendios (Bowman et al., 2011).

El colapso y la desestructuración de los sistemas socioeconómicos rurales mediterráneos a mediados del siglo XX supuso la despoblación de los espacios de interior y el abandono de los usos tradicionales del suelo. Esta pérdida de gestión y activos patrimoniales, unida a la aparición de nuevas dinámicas territoriales en relación con los procesos de urbanización, dio lugar a un aumento descontrolado del combustible y a la aparición de nuevos conflictos y realidades socioespaciales que se convirtieron en las fuerzas motrices de un nuevo régimen de fuego (San Miguel et al., 2017). Sin embargo, las causas de los incendios muestran marcadas inercias a escala local y reproducen patrones de ignición seculares relacionados con las características de los sistemas socioespaciales.

Partiendo de estas premisas, el fundamento teórico de la investigación llevada a cabo se apoya en tres bases principales: la teoría general de sistemas, la teoría de la resiliencia y la teoría cognitiva.

En realidad, la teoría de los sistemas socioecológicos es la referencia general del paradigma de los riesgos naturales en Geografía, que considera que el origen de las catástrofes se encuentra en el carácter de las relaciones que se establecen entre la población y sus entornos (O'Brien, O'Keefe, Gadema y Swords, 2010). Esta es la base teórica que se maneja generalmente en los trabajos sobre incendios forestales en ecosistemas mediterráneos para explicar la complejidad de las estructuras socioespaciales y su funcionamiento, que se encuentran en el origen de los factores de ignición y propagación del fuego (Martínez, Vega-García y Chuvieco, 2009).

En segundo lugar, la teoría de la resiliencia se ha utilizado también profusamente en las últimas cuatro décadas para explicar los cambios abruptos que acontecen en los sistemas socioecológicos (Garmestani,
Allen y Benson, 2013). La resiliencia es la capacidad de un sistema para asimilar un cambio y resistir el impacto de los riesgos que entraña (Adger, 2000). Los aspectos fundamentales de la teoría de la resiliencia son el papel determinante que juegan las dinámicas multiescalares y el desequilibrio de los sistemas socioecológicos. Recientemente, el concepto de resiliencia social y ecológica ha evolucionado desde la idea central de "resistir y recuperarse" hacia un planteamiento de "adaptación", y del objetivo de la "estabilidad" a la "realidad del cambio".

Por último, la teoría cognitiva examina los conceptos subyacentes a las acciones humanas, tales como los valores, las actitudes y las normas, y establece relaciones entre todas ellas (Czaja y Cottrell, 2014). Esta teoría se ha utilizado para interpretar las pautas de comportamiento social y los procesos de aprendizaje y de evolución cultural que permiten entender las causas de los incendios relacionadas con las actividades humanas a escala local. Se trata, en definitiva, de interpretar la vertiente social de los incendios forestales desde sus fundamentos primarios, y de entender los aspectos culturales del fuego y las buenas prácticas de su uso como herramienta de gestión del territorio (Montiel y Kraus, 2010).

\section{MATERIAL Y MÉTODOS}

\section{Fuentes para un análisis evolutivo de los incendios y el uso del fuego en el medio rural}

Desde 1968 España cuenta con una base de datos de incendios forestales, la Estadística General de Incendios Forestales (EGIF). Esta sistematización estadística a cargo del Ministerio de Agricultura respondía a la preocupante evolución del riesgo, con un agravamiento del problema de los incendios y una alarma social creciente (Carracedo, Diego, García y Rasilla, 2009; Vélez, 2009). Con desfases y diferencias regionales, se estaba produciendo una transición desde el régimen de fuego frecuente y de baja intensidad, en relación con su manejo como instrumento de gestión agraria, a la generalización del régimen de fuego catastrófico, con perímetros e intensidad cada vez mayores. Existe, sin embargo, una gran dificultad para valorar este cambio, ante el déficit de conocimiento del régimen de incendios durante el periodo anterior (Bouisset, 2007).

La evolución espaciotemporal de los incendios antes de 1968 había sido abordada a través de la información procedente de la administración forestal y mediante el recurso a la prensa escrita (Araque, 
1999; Pausas, 2004), en aproximaciones sesgadas hacia los incendios producidos en espacios arbolados y en los montes públicos. Una historia del régimen del fuego más amplia, basada en la búsqueda y análisis de fuentes documentales (judiciales, policiales, administrativas), hemerográficas y estadísticas ha sido iniciada para el Sistema Central, con la creación de una base de datos pionera en Europa: el Registro Histórico de Incendios Forestales (RHIF), que se remonta al año 1497 y cuenta con casi 7.000 registros de incendios históricos datados y documentados (Montiel, 2013a).

Para la provincia de Cáceres, el RHIF está alimentado principalmente, además de por el Ministerio de Agricultura y las fuentes hemerográficas, por la excepcional riqueza y calidad de la información procedente del Archivo Histórico Provincial (AHP). De aquí proceden numerosas referencias a incendios históricos extraídas de los fondos documentales de Real Audiencia, Juzgados de primera instancia e instrucción, Ayuntamientos, y Gobierno Civil (Araque et al., 2012).

Además del RHIF, la base del estudio la constituyen las respuestas recogidas en el Interrogatorio de la Real Audiencia de Extremadura de 1790, cuya documentación original se encuentra igualmente en el $\mathrm{AHP}^{2}$. Tras la creación de la provincia de Extremadura en 1785 , cinco años después se estableció en Cáceres la Real Audiencia, órgano jurisdiccional de la misma. Antes de que empezara a actuar, se dispuso la realización de un interrogatorio. Justicias, párrocos, ayuntamientos y particulares de los pueblos integrados en los partidos que formaban la Audiencia, previamente a las visitas realizadas a los partidos por los ministros, contestaron un cuestionario de 57 preguntas para facilitar el conocimiento del territorio (Rodríguez, 1981). Contiene, entre otros asuntos, información del mayor interés sobre los recursos municipales (propios del común), actividades agrícolas y ganaderas. Para el conocimiento del uso del fuego resultan especialmente útiles las siguientes preguntas:

"42. Si se reparten por suertes algunos montes á los vecinos para rozarlos, y en qué forma se practíca: si se perjudica a los árboles, ó se procuran conservar.

[...] 46. Si se suelen quemar los montes, y para qué fines: qué perjuicios se siguen de esto, y cómo se suele castigar este exceso" (Interrogatorio, 1993. Partido de Alcántara, pp. 73-74).
La prolija documentación judicial producida por la Real Audiencia documenta la conflictividad derivada de la presencia del fuego a través de numerosos litigios. Esta línea se ha continuado con los expedientes procedentes de los juzgados de primera instancia a partir de su creación en la primera mitad del siglo XIX, y en ella también confluye, en buena medida, la información administrativa procedente de algunos archivos municipales consultados ${ }^{3}$, especialmente las denuncias formuladas por la guardería rural. Por último, el fondo documental de Gobierno Civil del AHP de Cáceres sobre incendios forestales (comunicaciones remitidas por alcaldes y puestos de la Guardia Civil de ocurrencias de incendios en el medio rural) (Galiana, 2014) constituye una valiosa información para caracterizar y evaluar con precisión el régimen de incendios en los años previos a la serie estadística (1942-1966).

\section{Metodología}

El análisis crítico efectuado a partir de la consulta exhaustiva de las respuestas al Interrogatorio de la Real Audiencia de 1790 ha permitido realizar una primera caracterización del uso del fuego, poniéndolo en relación con su uso en diferentes prácticas agrarias y otorgándole una localización precisa a nivel de término municipal. También ha hecho posible valorar su consideración social, derivada de la conflictividad asociada al mismo, más allá de su carácter delictivo (Carmona, 2014). Siguiendo esta misma línea analítica, y con carácter complementario, se han manejado ordenanzas referentes al mismo asunto procedentes de comunidades de pasto y municipios.

En una segunda fase, se han determinado las permanencias y los cambios que experimenta el régimen de incendios durante el siglo XIX y primera mitad del siglo XX. La documentación judicial y administrativa consultada, y su rica información sobre la conflictividad asociada al uso del fuego, aporta valiosas conclusiones sobre las causas que están detrás de numerosos siniestros. En este mismo sentido, la caracterización y evaluación del régimen de incendios en los años previos al periodo de la serie estadística que se inicia en 1968, realizada con el fondo del Gobierno Civil del AHP entre 1942 y 1966 (Galiana, 2016), aporta sólidos argumentos para concluir acerca de las continuidades y los cambios en el uso del fuego, ilustrando además con valiosos ejemplos el nuevo régimen de incendios que se generaliza a partir de los años 1960. 


\section{EL USO DEL FUEGO A FINALES DEL SIGLO XVIII A PARTIR DE LAS RESPUESTAS AL INTERROGATORIO DE LA REAL AUDIENCIA DE 1790}

\section{Algunas consideraciones sobre la organización terri- torial y del terrazgo}

Las características del proceso colonizador y repoblador altomedieval son esenciales para entender el sistema de tenencia de la tierra a finales del siglo XVIII. Debe recordarse la importancia de las Comunidades de Villa y Tierra en este territorio que, junto a la Orden de Alcántara en su sector noroccidental, constituyeron la organización jurisdiccional básica de la actual provincia cacereña, implantando unos esquemas de distribución del espacio con un protagonismo casi absoluto de la propiedad concejil (Monsalvo, 2013; Diago, 2012). Hasta la desvinculación de su patrimonio en el siglo XIX, estas comunidades fueron dueñas de la mayor parte de la tierra. Ello explica la importancia de los bienes comunales y de propios en la organización del término durante el siglo XVIII, con los baldíos como parte más extensa del patrimonio concejil y comunal (Zulueta, 1975, 1977). Junto a las tierras concejiles, el predominio de la gran propiedad se completaba con las encomiendas de órdenes militares y las dehesas particulares.

Esta consolidación de la gran propiedad tenía en la dehesa su forma de explotación generalizada. En ella, el habitual arrendamiento para roza y siembra, y la labor como ocupación mayoritaria de un terrazgo dividido en hojas, fue perdiendo protagonismo frente a la dedicación exclusiva a pasto de buen número de dehesas a lo largo del siglo XVIII (Melón, 1989). La progresión de los pastos en detrimento de los cultivos, y su coincidencia con un significativo incremento demográfico a partir de 1770 (Zarandieta, Melón, Llopis, Rodríguez y Rodríguez, 1990), desencadenaron un hambre de tierras sin precedentes. Estos hechos están detrás de un intenso proceso roturador que, impulsado a su vez por la promulgación de normativas al efecto (Real Provisión de 2 de mayo de 1766, sobre el repartimiento de las tierras de labor de propios y baldíos concejiles entre senareros y braceros) (Melón, 1989), afectó de una manera creciente a tierras marginales ocupadas por el monte, propiciando, en consecuencia, la práctica generalizada de una agricultura de roza y quema sobre las mismas:

(Herreruela) "41. Como la escasez de tierra para la labor en este pueblo es tan grande, se entran los labradores en lo mas yntrincado de la sierra y en lo más aspero del rivero a desmontar para sembrarlo, sin embargo de que por costoso que le sea le precisan a pagar el diezmo y terrazgos como si fuera la mejor tierra"4 (Interrogatorio, 1993. Partido de Alcántara, p. 348).

\section{Rozas y quemados: el fuego en las prácticas agrícolas y ganaderas tradicionales (Fig. 3)}

La importancia histórica del uso del fuego en la provincia de Cáceres tiene que ver con dos hechos estrechamente relacionados. Por un lado, con la amplitud espacial e intensidad que alcanzaron los aprovechamientos ganaderos, desarrollados a partir de unas favorables condiciones agroclimáticas para los pastos de invernada; y por otro, con su coexistencia con una agricultura cerealícola adaptada a la pobreza edáfica de buena parte de su territorio. Esta coincidencia de usos se resolvió históricamente con el recurso a largos turnos de siembra en buena parte del terrazgo (Pereira, 1993), generando un espacio de cultivo temporal y rotatorio, con las rozas y quemas como práctica más frecuente en su preparación anual.

La roza y siembra, previo reparto de suertes entre los vecinos, se efectuaba preferentemente sobre los baldíos, aunque, en ocasiones, la amplitud del espacio montuoso apto para la roza ni siquiera precisaba del habitual sorteo. Igualmente se procedía en las encomiendas de la jurisdicción de la Orden de Alcántara. No fueron infrecuentes las roturaciones en dehesas concejiles (boyales, de propios), mediando el correspondiente reparto o subasta; más rara vez en terrenos particulares. La ejecución de rompimientos en contra de la voluntad de las Comunidades de villa y tierra no debía ser nada excepcional, aunque los testimonios que reconocen esta práctica ilícita sean escasos:

(Piornal) "41. Los mas de los terrenos que goza este Pueblo son incultos llenos de piedras, de mataraña, de roble y de berezo, ay muchos que a puro travajo y costa los rompen algunos pedazos y el no romper mas es por que lo impide la justizia temiendo la ciudad de Plasenzia y sino fuera por dichos Rompimientos no pudieran subsistir ni mantenerse sus habitadores, pues despues de la perdida de las castañas no se a esperimentado otro remedio mas util ni quemas, de forma que de impedir el cerrarlo se pierde esta población, cuios cierros son de parez y se cuida no impidan a los ganados, y son en nuestra propia deesa unos que hazen a tres celemines, otros a mas y menos. El no haver ojas ni rozas es por no poderse hazer como no sea de la forma que va referido" (Interrogatorio, 1993. Partido de Plasencia, p. 613). 
FIGURA 1

AVANCE DEL OLIVAR EN ANTIGUAS ROTURACIONES SOBRE “MATA PARDA” (LA PESGA)

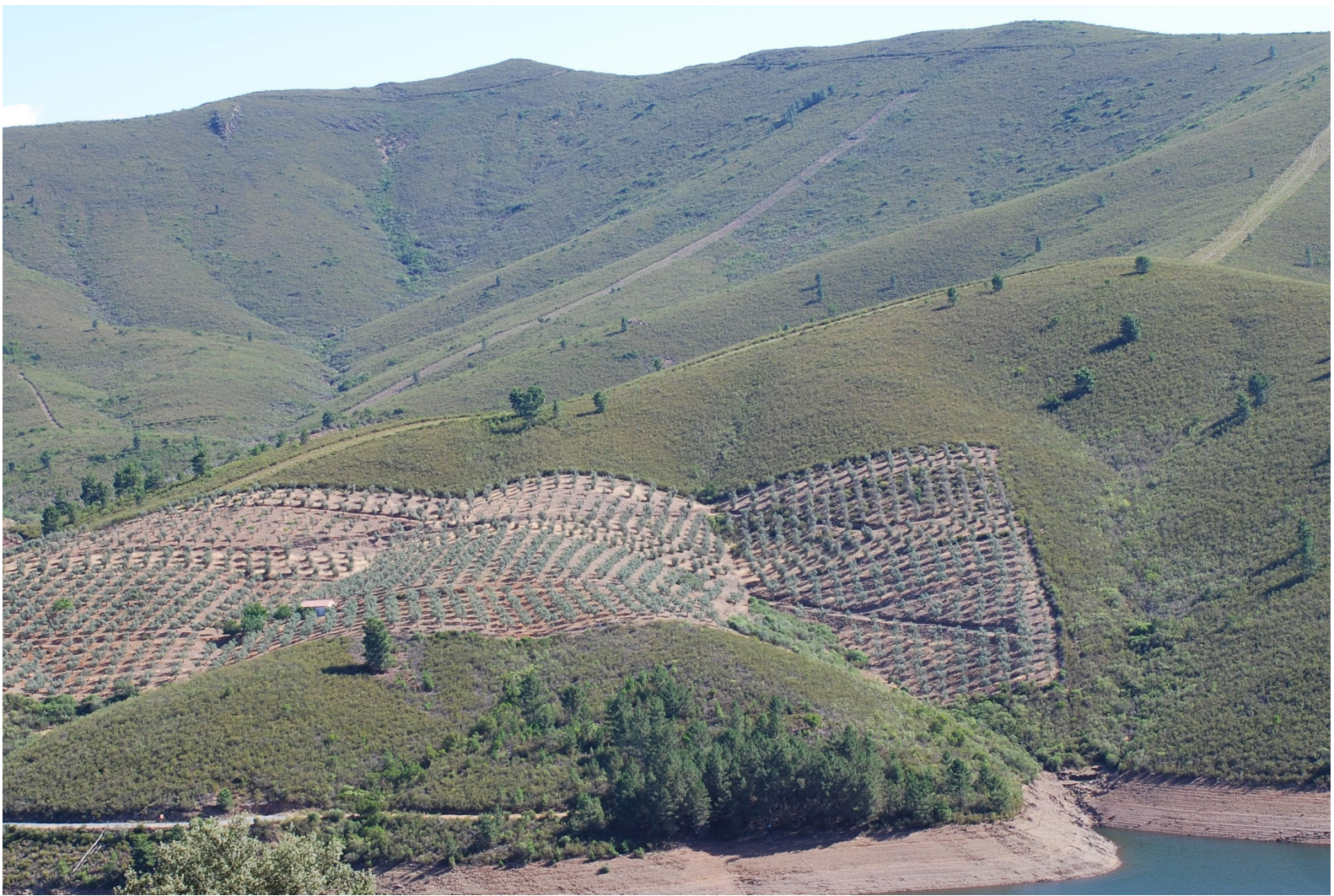

Era costumbre efectuar el sorteo el día de San Martín, adjudicando un número de suertes proporcional al número de yuntas, en un proceso perfectamente formalizado en todas sus fases (Rodríguez, 2000). La roza propiamente dicha (corta de la parte aérea de la vegetación arbustiva existente) se llevaba a cabo entre enero y marzo, antes de la formación de semillas, permaneciendo en el terreno hasta su quema en el rigor del estío; la sementera (centeno, trigo, cebada) se verificaba dos meses después (Zepeda, 1791). La acción de justicias y peritos velaba no sólo por la adecuada formación de las suertes, sino, sobre todo, por el modo en que debía prepararse el terreno para dar fuego a la roza, ejecutando las correspondientes rayas o aceros ${ }^{5}$, protegiendo de la quema el arbolado existente y aislándola del resto de monte. En el caso de que la roza se efectuara en una zona con arbolado, se procedía previamente a la poda de las ramas, apartando de los pies la leña rozada a una distancia de cuatro pasos (Zepeda, 1791).

Respetar el arbolado existente y evitar que el fuego se soltase eran las principales preocupaciones del proceso preparatorio a la quema:
(Mesas de Ibor) "42. Los montes que ay utiles para rozas se reparten por suertes medidas a varas con ygualdad, luego se hacen tantas voletas cuantos sujetos han pedido lavor, estas dobladas con el nombre de cada uno para dentro se meten en un cantaro. [...] Los montes que se parten para rozas no tienen arboles y caso que ayga alguno se ara, limpia y desbroza alrededor para su conservacion.

[...] 46. No se suelen quemar los montes en este termino, porque se echa buena raya a las rozas y se procura la orilla y ayre favorable al ponerlas fuego" (Interrogatorio, 1993. Partido de Plasencia, pp. 476-477).

(Villamesías) "42. Que se reparten por suertes a los vecinos los ejidos del termino cada tres años a tres hojas y que es preziso rozar la mata parda, pues si se deja crecer no se cojera grano y que aunque hay quema de la roza no se sigue perxuicio porque la separan de algun otro arbol si hay, y que es practica ynmemorial el laboreo de estos ejidos por no haber otras tierras en el termino" (Interrogatorio, 1993. Partido de Trujillo, tomo II pp. 884-885). 
En cualquier caso, la búsqueda del beneficio agrícola inmediato prevalecía sobre la conservación del arbolado en la quema del monte rozado, única forma de ampliar la superficie de cultivo ante el avance del uso privativo para pastos de buena parte de las dehesas. Pero, además, la baja calidad agrológica de estos terrenos marginales hacía del abonado mediante las cenizas el mejor y casi único modo de disponer convenientemente estas tierras para la cosecha, superando en efectividad al derrame de cal según extendida opinión de la época (Zepeda, 1791). Los turnos, en consecuencia, debían establecerse cuando el "monte pardo" (tierras montuosas llenas de arbustos) estuviera lo suficientemente "viejo" (apto para rozar), lo que podía demorarse entre doce y quince años. No obstante, las rozas con turnos más cortos eran frecuentes, ya por la presión de los labradores para disponer de tierras de cultivo en los baldíos, ya por la integración de esta práctica en la preparación de la hoja de cultivo en dehesas divididas en cuatro o cinco cuarteles.

(Alcuéscar) "[...] pues criandose y conservandose dichos montes pardos hasta hallarse sufizientes de doze o mas años, harian sus rozas los labradores y producirian (como la esperiencia lo acredita) a el duplo las mieses que en ella se sembrasen" (Interrogatorio, 1993. Partido de Mérida, p. 92).

(Toril) "42. En la dehesa y egido de esta villa hay quatro labores, cada año se saca una y se reparte por suertes entre los vezinos labradores para su sementera, es de poca roza con motivo de la continuación, y al tiempo de su quema se dan sus rayas correspondientes a los arboles y se practica la quema sin ofender al monte, el que se procura conservar conforme prevee la real ynstruccion, apostando y guiando anualmente por cima de dos mil chaparros" (Interrogatorio, 1993. Partido de Plasencia, p. 775).

Las operaciones de roza y quema tenían un modelo de referencia para el espacio forestal en su conjunto, que no era otro que la consecución de un monte ahuecado. La preparación del terreno para la siembra se debía acompañar de la entresaca y beneficio del arbolado productivo (encinas, alcornoques, quejigos), eliminando las matas inútiles (jara, brezo, lentisco, madroñera, rebolla, torvisco, aulaga) y favoreciendo además su uso ganadero:

(Majadas) "42. En todo el terreno desta villa hay en sitios vajos y en otros altos, se reparte por suertes a los vecinos para la lavor, de que unicamente viven, el monte bajo como jara, lentisca y otros semejantes se rozan y el alto como encinas, quejigos y robles se entresacan, y cuando se quema se procura guardar y guarda en lo posible el arbolado.
[...]. 44. El terreno desta villa está poblado como queda dicho de encinas, quejigos y robles por lo alto y por lo bajo de jara, lentisca, tomillo, verezo, retama, madroña, torvisca y aolaga; lo alto da vellota para la manutenzion del ganado de zerda y algunos alcornoques (aunque pocos) que dan corcha para las colmenas, y lo vajo solo sirve para beneficiar las tierras que se siembran con su zeniza por rozarse y quemarse antes" (Interrogatorio, 1993. Partido de Plasencia, pp. 445-446).

(Peraleda de la Mata) "42. [... ] que algunos montes bajos que ay pasan a los vecinos por suertes, a efecto de que quitando las matas que estan dehemas, dejen chaparros para que con el tiempo se ejecute un monte floreciente, lo que asi ejecutan labrandolas al mismo tiempo, con cuio veneficio se frutifica a los que quedan y estos abundan en crecer y embarnecer, de suerte que limpiados en poco tiempo se consigue el monte que ba esplicado" (Interrogatorio, 1993. Partido de Plasencia, p. 597).

Pero más allá de este beneficio ganadero añadido, las quemas en relación con esta actividad generaron otro tipo de prácticas completamente distintas a las asociadas a las rozas: los "quemados" o "quemorros" pastoriles (Abel, Hernández, López, Pulido y López, 2009). Las sierras cacereñas experimentaban con elevada frecuencia quemas atribuidas a los ganaderos del terreno dedicados al ganado menor (lanar y, sobre todo, cabrío), aunque no falten denuncias sobre la responsabilidad de los trashumantes foráneos por estas prácticas maliciosas realizadas en zonas llanas. Porque, en efecto, frente a la quema de rozas, altamente reglamentada como hemos podido comprobar, las prácticas pastoriles se ejecutaban desde la clandestinidad, con nocturnidad, por "sugetos ignorados", muy rara vez con licencia:

(Navasfrías) "46. Se experimentan quemas de montes, no se sabe por quienes, pero se presume sea por los pastores con el fin de quitar el abrigo a las fieras y criar nuevas yerbas, de lo cual se suele seguir perjuicio a los arboles, nunca se ha formado causa por ser imposible averiguar el agresor" (Interrogatorio, 1993. Anexo: Poblaciones disgregadas de Extremadura, p. 48).

(Gata) "46. Se queman los montes con fraude por sugetos ignorados, para el fin de que coma el ganado particularmente el cabrio, en grave perjuicio de la colmeneria, olibos, encina, roble y castaños que se queman" (Interrogatorio, 1993. Partido de Alcántara, p. 287).

(Partido de Trujillo) "Los montes de esta jurisdizion y partido están muy deteriorados, expecialmente los que corresponden a la subdelegación de Truxillo, [...] por el daño que causan con mano oculta los trasumantes en los fuegos que prenden para que con las 
quemas se mejore el pasto, lo que también executan los pastores de ganado cabrio, destruiendo por estos medios dichos montes sin castigarse los yncendiarios [...]" (Interrogatorio, 1993. Partido de Trujillo, tomo I pp. 60-61).

Este carácter clandestino, en el caso de los ganaderos trashumantes, podía esconder conflictos con la población local a la que se pretende excluir del uso de determinados espacios. Es lo que se denunciaba en la Tierra de Trujillo:

(Conquista) "46 y 47. Suelense quemar los montes o por fuegos sueltos, o por diligencias que hacen los trasumantes en sus posesiones para limpiarlas y exentar de arboles, pues de este modo no entra en sus dehesas ganado de zerda a la bellota, cuyo disfrute es de Truxillo y su tierra, cuyo gravamen sienten mucho [...]" (Interrogatorio, 1993. Partido de Trujillo, tomo I p. 489).

Pero, por lo general, los "quemados" extremeños responden a una de las finalidades más habituales en el uso del fuego pastoril: la eliminación de matorra- les para favorecer la salida de pasto más abundante y apetecible, de mayor calidad bromatológica (Lasanta, 2010). Junto a ello, el aumento de los recursos forrajeros se producía también mediante el "renuevo del monte", pues de esta manera era posible aprovechar los brotes tiernos. Además, se favorecía su apertura, permitiendo el tránsito de los ganados por el mismo y ahuyentando a posibles depredadores (lobo). Con respecto a la frecuencia del fuego, el monte se quemaba periódicamente, pero sin la regularidad y el método de la práctica de rozas. Agosto era el mes preferido para su realización, momento en el que todas las sierras experimentaban estas ocurrencias con mayor o menor extensión y violencia:

(Villanueva de la Sierra) "46. Algunos años en el estio suelen quemarse algunos pedazos de monte bajo como son de brezales, madroñeras, jaras y demas fusca, a causa de lumbres que echan maliciosamente y por lo regular suelen ser pastores, pero no se puede averiguar ni justificar el causante" (Interrogatorio, 1993. Partido de Coria, p. 505).

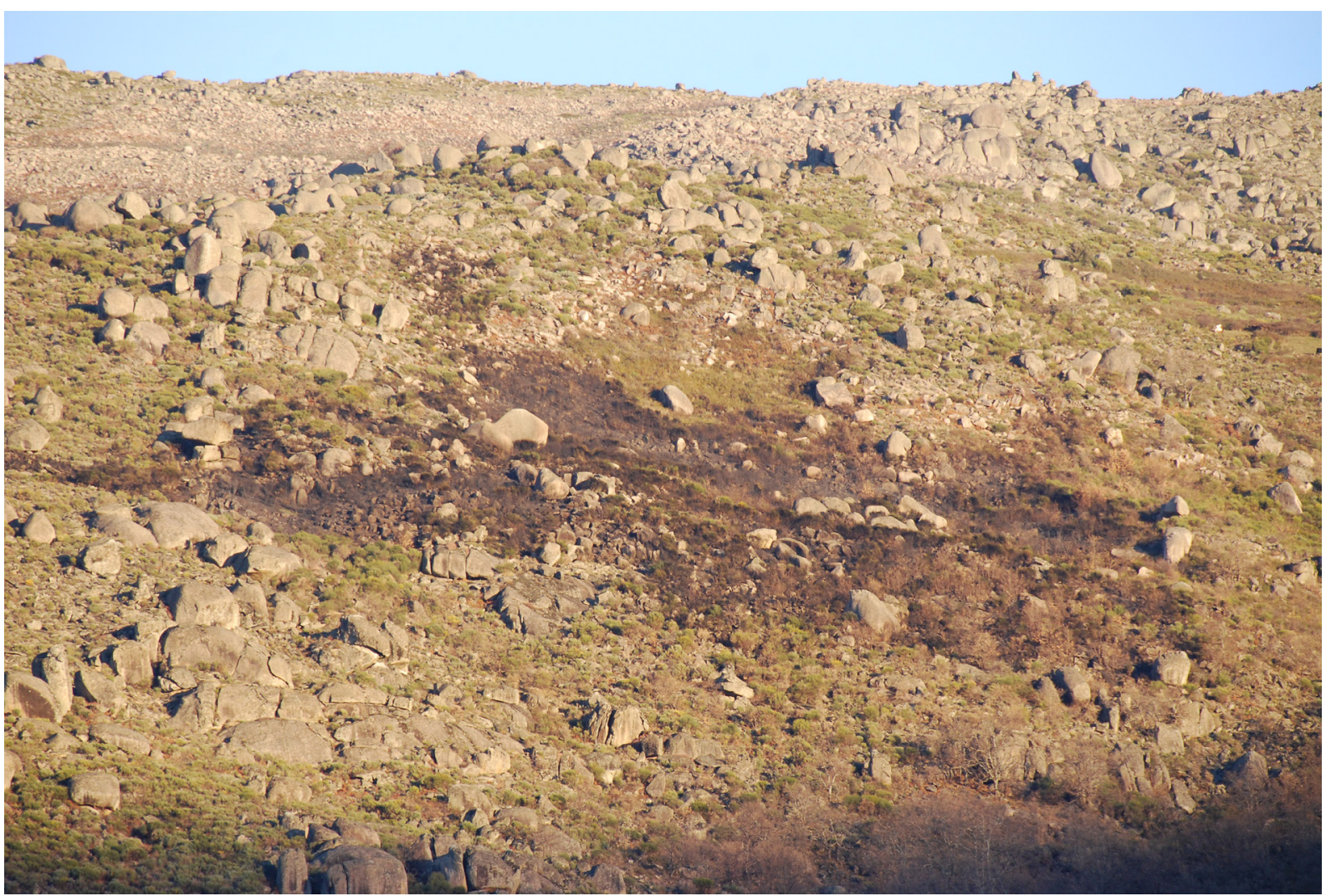


Por último, junto a las rozas y los fuegos pastoriles, también aparecen algunas referencias a la quema de montes para hacer carbón de brezo en Las Hurdes, Sierra de Gata, Sierra de San Pedro o Monfragüe. En 1945, el ingeniero de montes Butler, responsable de las repoblaciones forestales en Las Hurdes, describía así esta práctica, aún entonces vigente:

"Un aprovechamiento muy generalizado es el del carboneo, constituyendo una industria agotadora, y que se realiza quemando una gran extensión de terreno abundante en brezos y jaras, donde luego después de enfriado van generalmente las mujeres a extraer las cepas, haciendo con ellas el carbón" (Butler, 1945).

\section{Quemas e incendios: los conflictos asociados al uso del fuego}

A pesar del evidente y profuso uso útil del fuego, algunas de las respuestas siguen negando la existencia de estas prácticas, manifestando por el contrario la disposición del vecindario para hacer frente a algún posible incendio que se declarase:

(Jerte) "46. [...] los montes deste termino nunca se queman para fin alguno, antes vien se guardan y defienden con el maior cuidado de los incendios, y quando se orixina alguno acuden la xusticia con los vezinos y le apagan inmediatamente para evitar el perjuicio de los robles y castaños que se queman, y después dicha xusticia procede a la averiguazion y castigo del incendiario y a la exacción del daño causado" (Interrogatorio, 1993. Partido de Plasencia, p. 397).

Esta actitud de negación y respeto a la legalidad (Ordenanza de Montes y Plantíos de 1748) la encontramos exclusivamente en las zonas serranas, donde se concentran la mayor parte de los fuegos ganaderos de carácter clandestino. Los frecuentes incendios surgen de manera "inesperada", sin que "sea posible averiguar" quién puede estar detrás de los mismos, lo que obliga a la intervención de los vecinos:

(Garciaz) "46. Las quemas suelen ser mui comunes en los contornos, pero nunca he oido que se haia averiguado el incendiario, siguiendose a estos naturales mucha fatiga personal en apagar y luego detrimento para las vacas en la perdida de pastos. [...] Son frequentes las quemas, rara bez se averigua el yncendiario y causa mucho perjuicio a las abejas, ganados y arbolado" (Interrogatorio, 1993. Partido de Trujillo, tomo I pp. 704 y 709).

Frente al cainismo ante los ganaderos, la condescendencia hacia las prácticas agrícolas, que sólo daban lugar a accidentes inevitables:

(Logrosán) "46. En los montes confinantes a este termino se notan algunos años sus quemas, de modo que ia en monte de enzina o de jara o brezos, etc., se queman i deboran leguas de terreno, sin apearse quien sean los inzendiarios para su castigo, y solo quando de las labores de labradores se tiene la desgracia de que el fuego se les suelte sin poder contenerles, se procede contra tales labradores i pagan con el daño que resulta, sobre cuio particular los señores juezes conservadores miran estos lanzes con poca humanidad, pues deviendolos reputar por pura desgrazia y absolver de toda responsavilidad a dichos labradores como inculpables, exijen de estos considerables cantidades por via de denunzias, aunque les conte la inculpabilidad" (Interrogatorio, 1993. Partido de Trujillo, tomo II pp. 203-204).

$\mathrm{Y}$, sin embargo, buen número de respuestas en todos los ámbitos de la provincia coinciden en señalar que el problema de los incendios, y de los conflictos asociados al mismo, están provocados fundamentalmente por el fuego suelto de rozas:

(Partido de Trujillo) "Las rozas para sembrar también causan varios perjuicios con sus quemas por más precauciones que se tomen, [...] es muy frecuente salir el fuego de estas rozas y destruir más de dos leguas de dicho monte bajo con las colmenas y cuanto existe en su situación" (Interrogatorio, 1993. Partido de Trujillo, tomo I p. 61).

Por lo general, no se trataba de negligencias en la preparación del terreno y ejecución de la quema, sino las consecuencias del riesgo inherente asociado a una práctica extremadamente peligrosa. Las condiciones en las que se efectuaba la quema de la roza (en el centro del estío, con la vegetación arrancada completamente seca y esparcida por el terreno) favorecían la formación de torbellinos y la aparición de focos secundarios:

"[...] los torbellinos unas veces como he dicho levantan piedras, que revolviéndose unas contra otras, se quebrantan, cuyo ruido atemoriza; otras, y son las mas, levantan brasas y leños encendidos, que arrojan á gran distancia, como yo he visto haber saltado el tajo por tres partes, comunicando el fuego de una parte al monte de la orilla opuesta, y todos los días lo experimentan los Labradores, quando dan fuego á las rozas; pues á poco rato que comenzáron á encender, notan diferentes saltos y nuevas llamas, sin haber llegado allí el fuego" (Zepeda, 1791).

La proliferación de fuegos sueltos provocaba innumerables conflictos con otros aprovechamientos. Los daños más denunciados eran, sin lugar a dudas, los provocados a los corrales de colmenas, preferentemente localizados en las zonas serranas: 
(Alcuéscar) "Que la escasez de colmenas proviene la mayor parte de los continuados fuegos sueltos que todos los años se esperimentan, provenidos unos de no echar las suficientes raias a las rozas del monte pardo que hazen en estos terminos algunos labradores, tanto de esta villa como de la de Montánchez, y otros de los de este partido; y lo otro porque de intento los ponen en hora yntempestivas los cabreros por aprovechar con su ganado el retoño del monte pardo y cardillo; como tambien los carboneros para con mayor comodidad y menos trabajo hacer el carbon de brezo; [...]" (Interrogatorio, 1993. Partido de Mérida, p. 92).

Asistimos a un conflicto creciente, motivado por el auge de la labor en aquellos años, y que se enquistaba particularmente en ciertos ámbitos jurisdiccionales, como sucedía en Las Hurdes. En el municipio de Nuñomoral, por ejemplo, el reparto de aprovechamientos enfrentó secularmente a los habitantes del terrazgo hurdano (mayoritariamente cabreros y labradores) con los propietarios de los derechos sobre la cría de abejas, pertenecientes a la salmantina villa de La Alberca. En la Sierra de Gata, por su parte, las denuncias se planteaban en relación con los daños ocasionados a un olivar en expansión. En cualquier caso, y de manera habitual, la negativa consideración de la práctica de la roza se argumentaba en relación con los daños ocasionados al monte en general, y al arbolado de encinas, alcornoques y quejigos, en particular:

(Gata) "42. No se reparten los montes por suertes a los vezinos para rozarlos porque los que se le repartieron en la dehesa, ejido y Moeda del Fresno permanecen con ellos de efecto de Reales Ordenes, estos rozos se hacen con perjuicio del arbolado porque en el tiempo de su quema fenecen muchos pies y se dejan de criar otros, añadiendose a esto los cortes de los labradores" (Interrogatorio, 1993. Partido de Alcántara, p. 286).

(Villa del Campo) "42. [...] que los concegiles que tiene esta villa se reparten a los becinos por fanegadas para rozarlos y sembrarlos, en esta villa señor ya no hai un arbol casi pues con las rozas o no los tienen las suertes de tierra $(\mathrm{y})$ si los tienen son pocos y deteriorados, y las encinas y alcornoques van a finalizar enteramente en las tierras de lavor de este pueblo. [...]

46. [...] se queman los montes para la lavor de la tierra y sembrarla, los perjuicios que de las quemas resultan son no criarse un arbol de encina, alcornoque ni roble que produce este terreno; las quemas se hacen desolando, sin cuidado y con abandono del plantio si lo hai, no se cuida ni de encargar, castigar ni celar en quanto este punto" (Interrogatorio, 1993. Partido de Alcántara, pp. 663-664).
El monte que se quemaba (jara, brezo, lentisco, madroños, entre otros) era considerado "monte inútil", con nulos o muy bajos aprovechamientos. Sólo de manera excepcional encontramos testimonios que relacionaban el perjuicio ocasionado al monte, además de con la pérdida de aprovechamientos, con el deterioro de su función protectora:

(Casas de Millán) "46. Que alguna vez suelen enzenderse, sin saver quien, las sierras de este termino, por lo que no se puede castigar y los perjuizios que se siguen de esto son: el que en tiempo de lluvias suelen venir algunas avenidas que ocasionan ruinas en las paredes de los huertos y aun inundarse algunas casas" (Interrogatorio, 1993. Partido de Plasencia, p. 204).

Desde los pueblos no faltaron declaraciones que buscaban mejorar la coexistencia de la práctica de una agricultura de rozas con el resto de los aprovechamientos forestales. La vía de solución más frecuentemente propuesta, en este caso, era la de concentrar espacialmente las rozas, evitando su dispersión y el consiguiente incremento de los fuegos sueltos; igualmente se planteaba alargar su turno, favoreciendo con ello un pasto más abundante para las abejas:

(Partido de Trujillo) "Las rozas para sembrar también causan barios perxuicios con sus quemas por mas precauciones que tomen, pero obrando con arreglo no son las que mas dañan si estan en hojas continuadas, pero si las sacan en sitios discontinuos o separados, como en algunos valles que hay solo por exemplo quatro fanegas de terreno y doscientas de monte bajo ynutiles para sembrar, y asi en los demas es muy frecuente salir el fuego de estas rozas y destruir mas de dos leguas de dicho monte bajo con las colmenas y quanto existe en su situazion como lo he bisto, por lo que combendría prohibir semejantes arrompidos boluntarios y por lo regular executados sin las correspondientes licencias" (Interrogatorio, 1993. Partido de Trujillo, tomo I p. 61).

Sorprende encontrar ciertos testimonios de una evidente modernidad. Para ciertos montes, caracterizados por su fragosidad y aspereza, el fuego se consideraba la única vía para una gestión adecuada del mismo, contribuyendo a disminuir su vulnerabilidad frente a los incendios:

(Aldeanueva de la Vera) "45. [...] que en la dehesa de propios y boyal [...] ay algunos montes ympenetrables al ganado y solo sirben para el abrigo de las fieras, el qual fuera muy util desmontar y el medio por donde esto se puede conseguir es rozandolo para sembrar, y por el medio y temor de quemar tal qual arbol que ay entre dichos montes sin fructificar cosa alguna y es yndispensable sin embargo de tener mucho cuidado y 
hechar fuera de los arboles el monte para quemarlo aun mas de aquellos pasos que mandan las ordenanzas, se hallan estos terrenos perdidos y los arboles que en si tienen mucho mas y al fin suele venir una quema estrabiada y abrasarlos todos, por lo que combendría aunque se quemase alguno rozarlo y sembrarlo" (Interrogatorio, 1993. Partido de Plasencia, p. 54).

\section{Discusión. Permanencias y cambios en el uso del FUEGO DURANTE LOS SIGLOS XIX Y XX}

A fecha de 24 de febrero de 2016, el RHIF tenía referenciados, para la provincia de Cáceres, un total de 2.065 incendios ocurridos entre 1790, fecha del Interrogatorio de la Real Audiencia, y 1967, el año previo a la puesta en marcha de la Estadística General de Incendios Forestales del Ministerio de Agricultura. La información que lo nutre tiene diversas procedencias, entre las que destacan las búsquedas realizadas en hemeroteca (85 incendios); el archivo del Ministerio de Agricultura (218); el Boletín y estadísticas de la
Guardia Civil (137) y, sobre todo, el AHP de Cáceres (1.616). El número de referencias decrece conforme retrocedemos en el registro cronológico: 1.704 son del siglo XX, por 338 del XIX y 23 entre 1790 y 1800. Un volumen de información que permite extraer conclusiones bien fundamentadas.

En primer lugar, que las prácticas culturales de uso del fuego, tal y como fueron descritas a finales del siglo XVIII, pervivieron hasta los años 1960. Prueba de ello es que las negligencias en su uso constituyen la causa conocida más frecuente de incendio (447 registros) $)^{6}$. Aunque buen número de los incidentes registrados son fuegos sueltos, producto de las habituales labores de limpieza en el campo (quemas de zarzales, linderos, restos de poda), las rozas y quemas agrícolas, los fuegos ganaderos y el carboneo extensivo están en el origen de no pocos incidentes.

El carboneo de brezo siguió practicándose en las sierras de Gredos, Gata y Hurdes, prácticamente

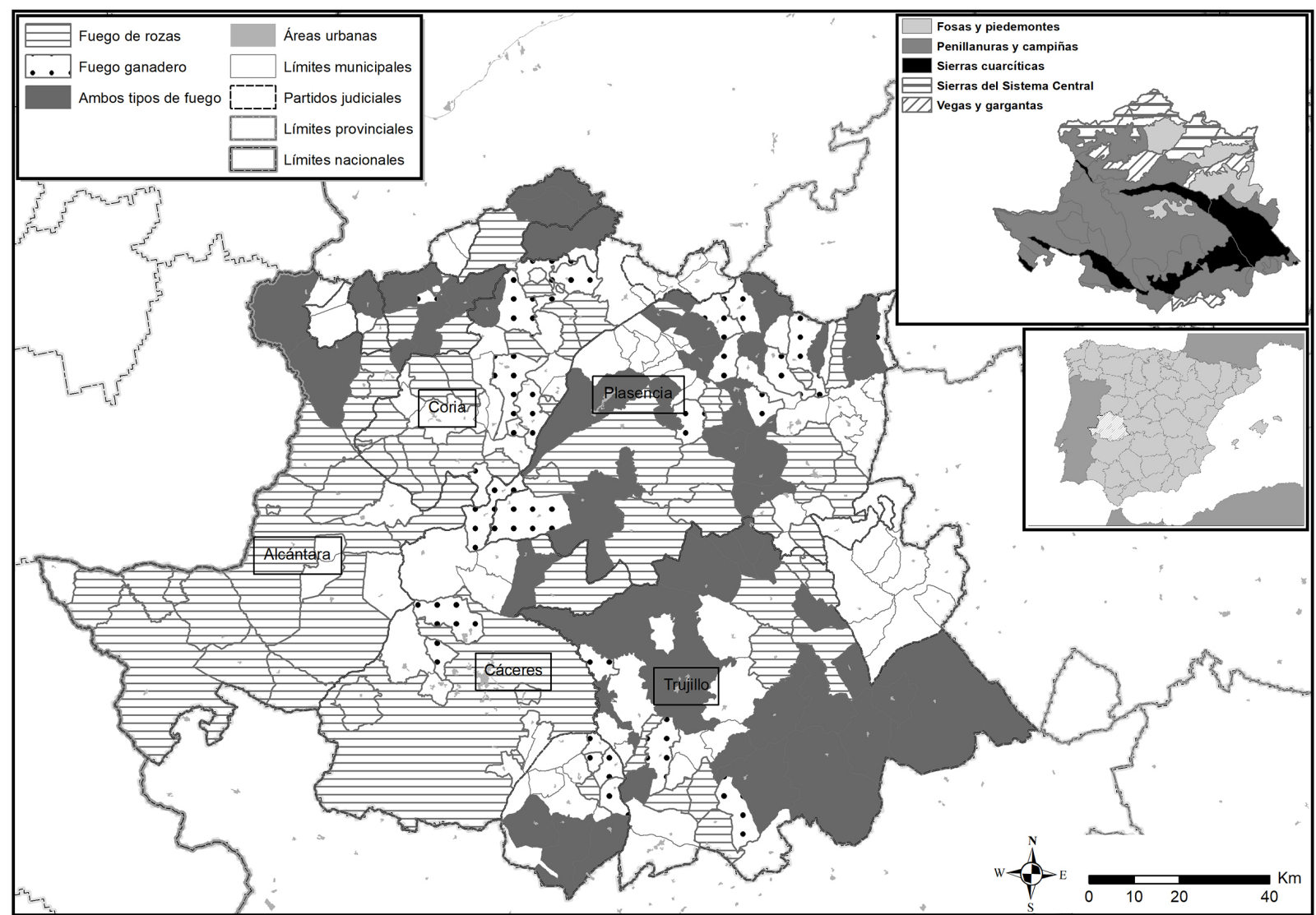

Fuente: Interrogatorio de la Real Audiencia (Interrogatorio, 1993). 
en cualquier época del año, provocando incendios que progresivamente constituyeron un elemento de alarma mayor por su afección a las incipientes repoblaciones forestales, lo que llevó a su acotamiento en ciertas zonas. La roza y quema de monte pardo, preparando el terreno para su posterior sembradura, causaron no pocos incidentes en toda la provincia, principalmente entre julio y septiembre, transgrediendo en muchos casos la normativa reguladora que intentaba trasladar estas prácticas al otoño, una vez recibidas las primeras Iluvias (Galiana, 2014). En ambos casos, desarrollos normativos tendentes a disminuir la peligrosidad de las prácticas, y cuya promulgación nos confirma su vigencia.

Pervivencias en las prácticas y continuidad de ciertas percepciones. Los fuegos ganaderos, y su propagación descontrolada por zonas no deseadas, no se consideraban negligencias, sino directamente fuegos intencionados. Especialmente durante los veranos, las zonas altas de las sierras de Gredos y Gata, sobre todo, continuaron siendo quemadas. La deplorable situación de las Hurdes a comienzos del siglo XX se achacaba a la reiteración del fuego provocado por los pastores (Araque, 1999). Y ya en época estadística, se seguía denunciando el fuerte componente de intencionalidad en la concentración de incendios producidos en los años 1970 en Gata-Hurdes y Vera-Tiétar (Alvarado, 1982), señalando directamente a los cabreros, y su voluntad de creación de pastos, como responsables de los mismos (ADENEX, 1999).

En definitiva, antes de la crisis rural de mitad del siglo XX, la evaluación de la presencia del fuego en el territorio no debe limitase a considerar la existencia de un régimen de incendios fuertemente controlado por la biomasa disponible y la población rural, caracterizado por pequeños incendios de vegetación (no únicamente forestales; también en el espacio agrícola), de baja intensidad y tamaño, frente a los que la defensa local (numerosa, experimentada y organizada) era perfectamente capaz de luchar con éxito (Galiana, 2014). También debe tener en cuenta la amplia presencia que todavía poseía la utilización del fuego útil.

La agudización del problema de los incendios se produjo en paralelo a la crisis del sistema tradicional agrario, con el éxodo rural como factor determinante. El cambio en el régimen de incendios que se documenta en la provincia de Cáceres a principio de los 60 es la expresión de una menor capacidad de gestión del territorio, y es también en parte el reflejo de una menor amplitud y recurrencia en el uso del fuego en la gestión territorial. Para determinados paisajes, como hemos tenido ocasión de comprobar, el fuego era un componente fundamental en su configuración y mantenimiento. El déficit de fuego (Pausas, 2004) que dichos paisajes fueron acumulando a partir del abandono de su práctica, contribuyó a un incremento de su vulnerabilidad y a la disminución de su resiliencia.

\section{CONCLUSIONES}

El manejo de documentación histórica de procedencia diversa y amplio rango temporal (siglos XVIII, $X I X$ y XX) ha permitido realizar una rigurosa caracterización de la importancia que tuvo el uso del fuego en el marco de los sistemas agrarios tradicionales mediterráneos, y de su evolución hasta la crisis rural de mediados de la pasada centuria. El ejemplo de la provincia de Cáceres es particularmente expresivo al respecto, dada la amplitud e intensidad que el uso del fuego llegó a alcanzar en las prácticas agroganaderas en este territorio.

La búsqueda de referencias históricas sobre la presencia del fuego presenta no pocas dificultades. La principal de ellas deriva de su cotidianeidad. La normalidad y frecuencia del uso del fuego en numerosas prácticas agrícolas y ganaderas provoca que las noticias sobre el mismo provengan, casi exclusivamente, de situaciones excepcionales, en los que su uso genere daños o conflictos. Denuncias, pleitos, actuaciones policiales y judiciales se convierten en las vías de acceso a una realidad evidente y oculta a un tiempo, mucho más amplia que lo que la estricta documentación nos informa. Relaciones territoriales que entren en el detalle de la descripción del uso del fuego, como es el caso del Interrogatorio de la Real Audiencia, son absolutamente excepcionales.

La voluntad de llegar a un conocimiento profundo de la presencia histórica del fuego en el territorio, complementando las aproximaciones al mismo a partir de fuentes estadísticas, está en el origen del RHIF. La base de datos nacional de incendios se inicia, y con grandes limitaciones, a partir de 1968, cuando los incendios se han convertido en un elemento de alarma social y política, cuando su comportamiento ya ha cambiado de manera súbita. Para ilustrar ese cambio es imprescindible un conocimiento de la realidad anterior que permita establecer relaciones explicativas útiles, contribuyendo a una gestión de este riesgo mejor informada. 


\section{AgRAdECIMIENTOS}

Nuestro agradecimiento a los miembros del Grupo de Investigación UCM-930329 “Geografía, Política y Socioeconomía Forestal”, por su contribución a organizar la base de datos del RHIF, y en particular a Oskar Karlsson, por su colaboración en la figura 3.Esta investigación se ha desarrollado en el Grupo

\section{NOTAS}

1. El régimen de fuego se define en función de la intensidad, estacionalidad, frecuencia, tipo (de superficie o de copas) y patrones espaciales de los incendios.

2. Interrogatorio formado de orden del Consejo para el gobierno del Regente y Ministros de la Real Audiencia de Extremadura, en la Visita que deben practicar en los partidos de aquella Provincia que se les han asignado por el Excmo. Sr. Conde de Campomanes, Gobernador del Consejo. 1790. Reproducido íntegramente en 1993, en edición de la Asamblea de Extremadura (Interrogatorio, 1993).

\section{BibLIOgRAFíA}

Abel Schaad, D., Hernández Carretero, A., López Merino, L., Pulido Díaz, F. y López Sáez, J.A. (2009). Cabras y quemorros: tres siglos de cambios en el paisaje de la vertiente extremeña de la Sierra de Gredos. Revista de Estudios Extremeños, LXV(1), 449-478.

Abel-Schaad, D. y López-Sáez, J.A. (2013). Vegetation changes in relation to fire history and human activities at the Peña Negra mire (Bejar Range, Iberian Central Mountain System, Spain) during the past 4,000 years. Vegetation history and archaeobotany, 22(3), 199-214.

ADENEX (1999). Incendios y cerezos ganan terrenos a los melojares del norte de Cáceres. Quercus, 165, 52-53.

Adger, W.N. (2000). Social and ecological resilience: are they related?. Progress in Human Geography, 24(3), 347-364.

Alvarado Corrales, E. J. (1982). Distribución espacial de los incendios forestales en Cáceres. Norba. Revista de arte, geografía e historia, 3, 77-88.

Araque Jiménez, E. (Ed.) (1999). Incendios históricos: una aproximación multidisciplinar. Baeza, España: Universidad Internacional de Andalucía. de Investigación Complutense "Geografía, Política y Socioeconomía Forestal" y ha sido financiada por el Ministerio de Economía y Competitividad en el marco en el marco de los Proyectos Nacionales I+D+i GEOINFOR (CSO2010-21788-C02-01), FIRESCAPE (CSO2013-44144-P) y FIRESCENARIOS (CSO2017-87614-P).

3. Serradillas, Nuñomoral.

4. Las citas textuales del Interrogatorio de la Real Audiencia de 1790 se efectúan a partir de la transcripción editada por la Asamblea de Extremadura (ver nota 2). Se informa del municipio (entre paréntesis), y se referencia el volumen del Partido y página.

5. Fajas de anchura limitada intensamente desbrozadas. Se practicaban delimitando la roza y rodeando el arbolado incluido en la misma.

6. Un buen número de los incendios registrados en el periodo de estudio son de origen casual.

Araque Jiménez, E., Galiana Martín, L. y Montiel Molina, C. (2012). Incendios forestales históricos en el Sistema Central, siglos XIX y XX. Primera aproximación. En R. Baena et al. (Coords.). Investigando en rural (pp. 289-296). Sevilla, España: Ulzama Ediciones.

Balboa López, X. (1999). El fuego en la historia de los montes gallegos: de las rozas al incendio forestal. En E. Araque (Ed.). Incendios históricos: una aproximación multidisciplinar (255-276). Baeza, España: Universidad Internacional de Andalucía.

Barbier, E. B., Burgess, J. C. y Grainger, A. (2010). The forest transition: Towards a more comprehensive theoretical framework. Land use policy, 27(2), 98-107.

Berrogain, G. (1930). Ordenanzas de La Alberca y sus términos Las Hurdes y Las Batuecas. Anuario de historia del derecho español, 7, 381-441.

Blanco Carrasco, J.P. (1994). Una visión inédita de las Hurdes en el siglo XVIII. Alcántara: revista del Seminario de Estudios Cacereños, 31, 161-192.

Bouisset, C. (2007). La protection des fôrets contre l'incendie: d'une affaire locale et privée à un problème départemental: l'exemple des Pyrénées- 
Orientales (1920-1970). Sud-ouest européen: revue géographique des Pyrénées et du Sud-Ouest, $23,79-88$.

Bowman, D. M. J. S., Balch, J., Artaxo, P., Bond, W. J., Cochrane, M. A., D'Antonio, C. M., DeFries, R., Johnston, F. H., Keeley, J. E., Krawchuk, M. A., Kull, C. A., Mack, M., Moritz, M. A., Pyne, S., Roos, C. I., Scott, A. C., Sodhi, N. S., Swetnam, T. W. y Whittaker, R. (2011). The human dimension of fire regimes on Earth. Journal of Biogeography, 38(12), 2.223-2.236.

Butler, J.M. (1945). Las Hurdes y sus problemas. II. Revista Montes, (3), 111-125.

Carmona Gutiérrez, J. (2014). El delito en el campo: daños, hurtos, fuegos y cortes en el ámbito rural extremeño a finales del Antiguo Régimen. Clio \& Crimen: Revista del Centro de Historia del Crimen de Durango, 11, 79-100.

Carracedo, V., Diego, C, García Codrón, J.C y Rasilla, D.F. (2009). Los incendios forestales. Barcelona, España: Davinci Continental.

Czaja, N. y Cottrell, S.P. (2014). Integrating social science research into wildland fire management. Disaster prevention and management, 23(4), 381-394.

Diago Hernando, M. (2012). Los aprovechamientos de las tierras de titularidad pública en las Comunidades de Villa y Tierra de la Extremadura castellano-leonesa entre los ss. XIII y XVII. En V. Muñoz Gómez (Coord.). Las Comunidades de Villa y Tierra: dinámicas históricas y problemáticas actuales (85-113). Murcia, España: Editum.

Galiana Martín, L. (2014). Régimen de incendios y crisis del sistema rural en la zona occidental del sistema central (Provincia de Cáceres) (1940-1968). En J. Olcina y A. Rico (Coords.). Libro jubilar en homenaje al profesor Antonio Gil Olcina (571-588). Alicante, España: Universidad de Alicante, Instituto Interuniversitario de Geografía.

Galiana Martín, L. (2016). Incendios forestales y crisis rural en el norte de Cáceres (1940-1968): Nuevas fuentes para su estudio. Revista Montes, 125, 24-30.

García Pérez, J. y Sánchez Marroyo, F. (1984). Extremadura a fines del s. XVIII y comienzos del XIX: conflictos campesinos, crisis agrarias, crisis de subsistencia y agobios fiscales. Norba. Revista de historia, 5, 213-234.

Garmestani, A.S., Allen, C.R. y Benson, M.H. (2013). Can law foster social-ecological resilience?. Ecology and Society, 18(2), 37.
Interrogatorio (1993). Interrogatorio de la Real Audiencia. Extremadura a finales de los tiempos modernos (11 vols). Mérida, España: Asamblea de Extremadura.

Iriarte-Goñi, I. y Ayuda, M.I. (2018). Should Forest Transition Theory include effects on forest fires? The case of Spain in the second half of the twentieth century. Land Use Policy, 76, 789-797.

Krebs, P., Pezzatti, G.B., Mazzoleni, S., Talbot, L.M. y Conedera, M. (2010). Fire regime: history and definition of a key concept in disturbance ecology. Theory in Biosciences, 129(1), 53-69.

Lasanta Martínez, T. (2010). Pastoreo en áreas de montaña: Estrategias e impactos en el territorio. Estudios geográficos, 71(268), 203-233.

Lázaro, A. y Montiel, C. (2010). Overview of prescribed burning policies and practices in Europe and other countries. En J.S. Silva, F. Rego, E. Rigolot y P. Fernandes (Eds.). Towards integrated fire management - outcomes of the European project Fire Paradox (137-150). Joensuu, Finland: European Forest Institute. EFI Research Report 23,137-150.

Leone, V. (1999). Los incendios en el Mediodía italiano. En E. Araque (Ed.). Incendios históricos: una aproximación multidisciplinar (219-249). Baeza, España: Universidad Internacional de Andalucía.

Martínez, J., Vega-García, C y Chuvieco, E. (2009). Human-caused wildfire risk rating for prevention planning in Spain. Journal of Environmental Management, 90: 1.241-1.252.

Mather, A.S. (1992). The forest transition. Area, 24(4), 367-379.

Melón Jiménez, M.Á. (1989). Extremadura en el Antiguo Régimen: Economía y Sociedad en tierras de Cáceres, 1700-1814. Mérida, España: Editora Regional de Extremadura.

Monsalvo Antón, J.M. (2013). Frontera pionera, monarquía en expansión y formación de los concejos de Villa y Tierra: Relaciones de poder en el realengo concejil entre el Duero y la cuenca del Tajo (c. 1072-c. 1222). Arqueología y territorio medieval, 10 (2), 45-126.

Montiel Molina, C. (2013a). Investigación geohistórica sobre las causas de los incendios forestales. Revista Montes, 114, 17-21.

Montiel Molina, C. (Coord.) (2013b). Presencia histórica del fuego en el territorio. Madrid, España: Ministerio de Agricultura, Alimentación y Medio Ambiente. 
Montiel, C. y Kraus, D.T. (Eds.) (2010). Best Practices of Fire Use: Prescribed Burning and Suppression Fire Programmes in Selected Case-study Regions in Europe. Joensuu, Finland: European Forest Institute. Research Report, 24.

Naveh, Z. (1975). The evolutionary significance of fire in the Mediterranean region. Vegetatio, 29(3), 199-208.

O’Brien, G., O'Keefe, Ph., Gadema, Z. y Swords, J. (2010). Approaching disaster management through social learning. Disaster Prevention and Management: An International Journal, 19(4), 498-508.

Pasquale, G.D., Martino, P.D. y Mazzoleni, S. (2004). Forest history in the Mediterranean region. En S. Mazzoleni, G. di Pasquale, M. Mulligan, P. di Martino y F. Rego (Eds.). Recent dynamics of the Mediterranean vegetation and landscape (13-20). New Jersey, USA: John Wiley \& Sons.

Pausas, J.G. (2004). Changes in fire and climate in the eastern Iberian Peninsula (Mediterranean basin). Climatic Change, 63(3), 337-350.

Pausas, J.G. y Keeley, J.E. (2009). A Burning Story: The Role of Fire in the History of Life. Bioscience, 59(7), 593-601.

Pausas, J. y Fernández, S. (2012). Fire regime changes in the Western Mediterranean Bassin: from fuel limited to drough driven fire regime. Climatic Change, 110, 2.115-2.226.

Pereira Iglesias, J.L. (1993). Organización, dedicación y explotación del espacio agrario en la Extremadura del Antiguo Régimen. Mélanges de la Casa de Velázquez, 29(2), 231-251.

Pyne, S.J. (2012). Vestal fire: an environmental history, told through fire, of Europe and Europe's encounter with the world. Washington, USA: University of Washington Press.

Rodríguez Cancho, M. (1981). Interrogatorios del siglo XVIII. Estudio comparativo. Norba. Revista de arte, geografía e historia, 2, 221-232.

Rodríguez Grajera, A. (2000). Las Ordenanzas Locales como fuente para la Historia Ambiental durante el Antiguo Régimen en Extremadura. Chronica nova:
Revista de historia moderna de la Universidad de Granada, 27, 167-197.

Rudel, T.K., Schneider, L. y Uriarte, M. (2010). Forest transitions: An introduction. Land Use Policy, 27(2), 95-97.

San Miguel, J., Chuvieco, E. Handmer, J., Moffat, A. Montiel, C., Sandahi, L. y Viegas, D. (2017). Climatological risk: wildfires. En K. Poljansek, M. Martin Ferrer, T. De Groeve and I. Clark (Eds.). Science for disaster risk management 2017: knowing better and losing less (294-305). Luxembourg: Publications Office of the European Union.

Sánchez Martínez, J.D., Araque Jiménez, E., Pulido Mérida, R. y Moya García, E. (1999). Los incendios forestales en Andalucía y Extremadura durante el tránsito de los siglos XIX al XX. En E. Araque Jiménez (Ed.). Incendios históricos: una aproximación multidisciplinar (163-214). Baeza, España: Universidad Internacional de Andalucía.

Turco, M., Bedía, J., Di Liberto, F., Fiorucci, P, von Hardenberg, J., Koutsias, N., Llasat, M.C., Xystrakis, F. y Provenzale, A. (2016). Decreasing fires in Mediterranean. PLoS ONE, 11(3), e0150663.

Vélez, R. (ed.) (2009). La defensa contra incendios forestales. Fundamentos y experiencias. Madrid, España: McGraw-Hill.

Zarandieta Arenas, F., Melón Jiménez, M.Á, Llopis Agelán, E., Rodríguez Cancho, M. y Rodríguez Grajera, A. (1990). El movimiento de la población extremeña durante el Antiguo Régimen. Revista de Historia Económica - Journal of Iberian and Latin American Economic History, 8(2), 419-464.

Zepeda y Vivero, J.A. (1791). Agricultura metódica, acomodada a la práctica del país, con varias noticias acerca de la naturaleza, propagación y extinción de la langosta. Madrid, España: Oficina de Don Benito Cano.

Zulueta Artaloytia, J. A. (1975). La venta de bienes comunales y concejiles en la Tierra de Cáceres. Estudios geográficos, 140-141, 1.159-1.188.

Zulueta Artaloytia, J.A. (1977). La tierra de Cáceres. Estudio Geográfico. Madrid, España: Instituto Juan Sebastián Elcano. 